\title{
Solution of the Neumann Problem with Respect to the Equation for Gravity-Gyroscopic Waves by the Finite Element Method
}

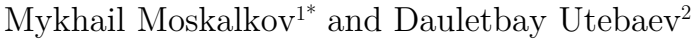 \\ ${ }^{1}$ National Aviation University, Kyiv, Ukraine \\ ${ }^{2}$ Berdakh Kara-Kalpak State University, Nukus, Uzbekistan \\ Email: moskalkov@hotmail.com
}

\begin{abstract}
In this paper, on the basis of a numerical finite element method, the solution of the Neumann problem with respect to the oscillation equation for gravity-gyroscopic waves is discussed. The approximation with respect to spatial variables is achieved by using linear splines, and the approximation with respect to time is achieved by using cubic Hermitean splines. It is demonstrated that the use of such approximation with respect to time allows the quality of the solution to be essentially improved as compared with the traditional approximation ensuring the second order accuracy. The stability and accuracy of the method are estimated. Using the method of regularization with spectrum shift, a new method is developed for solving the spatial operator degeneration problem associated with the Neumann problem. The results of the numerical calculations performed provide the possibility to make conclusions on the mode of behavior of the solution of the Neumann problem depending on the problem variables.
\end{abstract}

Keywords: Finite Element Method, Difference Scheme, Errors Estimates, Gravity-Gyroscopic Waves.

\section{Some Designations:}

$>u(x, t) \in H=\stackrel{0}{W}_{2}^{1}(\Omega)$ is the general solution of a differential problem;

$>u(t) \in H$ is the same solution expressed as an abstract function of argument $t$ with values in $H$;

$>u_{h}(t) \in H_{h} \subset H$ is the abstract function of continuous argument $t$ with values in $H_{h}$ which is obtained by the approximation of $u(t)$ by spatial variables;

$y=y^{n}=y\left(t_{n}\right), \dot{y}=\dot{y}^{n}=\frac{d y}{d t}\left(t_{n}\right), \hat{y}=y^{n+1}=\left(t_{n+1}\right), \hat{\dot{y}}==\dot{y}^{n+1}=\frac{d y}{d t}\left(t_{n+1}\right)$ is the approximate solution and its time derivative at the instants of time $t_{n}$ and $t_{n+1}$;

$\phi(\xi)=\left\{\begin{array}{ll}1-|\xi|, & |\xi| \leq 1 \\ 0, & |\xi|>1\end{array}\right.$ is the Kurant piecewise linear function.

\section{Statement of the Problem}

Let us consider the Neumann problem with respect to the equation for gravity-gyroscopic waves [1]:

$$
\begin{gathered}
\frac{\partial^{2}}{\partial t^{2}}(L u)+\omega_{0}^{2} L_{1} u+\alpha^{2} L_{3} u=-f(x, t),(x, t) \in Q_{T}=\left\{x=\left(x_{1}, x_{3}\right) \in \Omega \subset R^{2}, t \in(0, T]\right\} \\
\frac{\partial u}{\partial n}=0, x \in \Gamma=\partial \Omega, t \in(0, T]
\end{gathered}
$$




$$
u(x, 0)=u_{0}(x), \frac{\partial u}{\partial t}(x, 0)=u_{1}(x), x \in \bar{\Omega}
$$

where $L u=\frac{\partial^{2} u}{\partial x_{1}^{2}}+\frac{\partial^{2} u}{\partial x_{3}^{2}}, L_{m} u=\frac{\partial^{2} u}{\partial x_{m}^{2}}, m=1.3$, and $\frac{\partial u}{\partial n}$ is the derivative with respect to a normal to boundary $\Gamma$ of area $\bar{\Omega}=\left\{0 \leq x_{m} \leq l_{m}, m=1.3\right\}$.

Equation (1) is derived from the system of hydrodynamic equations according to the Bussinesk approximation [1-5]. This equation describes small-amplitude oscillations of stratified liquid with density $\rho_{0}\left(x_{3}\right)$, rotating about axis ox with duplicated rotation frequency $\alpha$. The squared value of VeisyalBrent frequency is designated as $\omega_{0}^{2}\left(x_{3}\right)=-\frac{\rho_{0}^{\prime}\left(x_{3}\right)}{\rho_{0}\left(x_{3}\right)}$. For simplification, value $\omega_{0}$ is considered as a constant one. The velocity of liquid is determined as $\vec{v}=\left(\frac{\partial u}{\partial x_{3}},-\frac{\partial u}{\partial x_{1}}\right)$. Equation (1) is neoclassical and belongs to Sobolyev equations of composite type [1-3].

In terms of hydrodynamics, Neumann boundary conditions (2) correspond to no-flow of liquid through boundary $\Gamma$. In our paper [6], Dirichlet conditions $u=0, x \in \Gamma$, which correspond to free flow of liquid through the boundary, were assumed [1].

Let us determine the general solution of problem (1-3) as function $u(x, t) \in H=W_{2}^{1}(\Omega)$, which for every $t \in[0, T]$ has derivative $\frac{\partial^{2} u}{\partial t^{2}} \in W_{2}^{1}(\Omega)$, and for $t \in(0, T]$ meets the following conditions:

$$
a\left(\frac{d^{2} u}{d t^{2}}(t), \vartheta\right)+\omega_{0}^{2} a_{1}(u(t), \vartheta)+\alpha^{2} a_{3}(u(t), \vartheta)=(f(t), \vartheta), \forall \vartheta \in H, u(0)=u_{0}, \frac{d u}{d t}(0)=u_{1}
$$

where

$$
a_{m}(u, \vartheta)=\iint_{\Omega} \frac{\partial u}{\partial x_{m}} \frac{\partial \vartheta}{\partial x_{m}} d x, m=1,3, \forall \vartheta \in H, a(\vartheta, \vartheta)=\sum_{m=1}^{2} a_{m}(\vartheta, \vartheta)
$$

and $u(t)$ is a function of abstract argument $t \in[0, T]$ with values in $H$. The existence of a solution and its properties are discussed in papers [1-3].

\section{Spatial Approximation}

Let $u s$ introduce in area $\bar{\Omega}$ net $\bar{\omega}_{h}=\bar{\omega}_{h_{1}} \times \bar{\omega}_{h_{3}}$, where $\bar{\omega}_{h_{m}}=\left\{x_{m}=x_{i_{m}}=i_{m} h_{m}, i_{m}=\overline{0, N_{m}}\right.$, $\left.h_{m}=l_{m} / N_{m}\right\}, m=1.3$. Let us create subspace $H_{h} \subset H$ by using the finite element method $[7,8]$ and the following basis:

$$
\left\{\phi_{i_{1} i_{3}}\left(x_{1}, x_{3}\right)=\phi\left(\frac{x_{1}-x_{i_{1}}}{h_{1}}\right) \phi\left(\frac{x_{3}-x_{i_{3}}}{h_{3}}\right), i_{1}=\overline{0, N_{1}}, i_{3}=\overline{0, N_{3}}\right\},
$$

where $\phi(\xi)$ is the known Current piecewise linear function.

The approximation for solution $u\left(x_{1}, x_{3}, t\right)$ is to be determined as follows:

$$
u_{h}\left(x_{1}, x_{3}, t\right)=\sum_{i_{1}=0}^{N_{1}} \sum_{i_{3}=0}^{N_{3}} h_{1} h_{3} u_{h}\left(x_{i_{1}}, x_{i_{3}}, t\right) \phi\left(\frac{x_{1}-x_{i_{1}}}{h_{1}}\right) \phi\left(\frac{x_{3}-x_{i_{3}}}{h_{3}}\right) \in H_{h} .
$$

On replacing the exact solution in (4) with the corresponding approximation, the approximate finiteelement solution is determined from (4) as follows: 


$$
\begin{gathered}
a\left(\frac{d^{2} u_{h}}{d t^{2}}(t), \vartheta\right)+\omega_{0}^{2} a_{1}\left(u_{h}(t), \vartheta\right)+\alpha^{2} a_{3}\left(u_{h}(t), \vartheta\right)=\left(f_{h}(t), \vartheta\right), \quad \forall \vartheta \in H_{h}, \\
u_{h}(0)=u_{0, h}, \frac{d u_{h}}{d t}(0)=u_{1, h} .
\end{gathered}
$$

Definition (5) is in agreement with the following Cauchy problem for function $u_{h}(t) \in H_{h}$ :

$$
D \frac{d^{2} u_{h}(t)}{d t^{2}}+A u_{h}(t)=f_{h}(t), u_{h}(0)=u_{0, h}, \frac{d u_{h}}{d t}(0)=u_{1, h}
$$

where

$$
D \equiv A_{1}+A_{2}=D^{*} \geq 0, A \equiv \omega_{0}^{2} A_{1}+\alpha^{2} A_{2}=A^{*} \geq 0
$$

The differences of the approximate solution of the problem (1-3) from the solution in our paper [6] are the following:

a) The use of boundary conditions (2).

b) The approximation with respect to spatial variables (in [6] a net method is used). As a result, the template for the internal net points consists of 9 nodes instead of 5 nodes in [6].

c) Matrix operators $D, A$ are degenerate operators, that is, zero eigenvalues $\lambda_{D}=0, \lambda_{A}=0$ correspond to zero eigenvector $v \equiv 1$.

\section{Approximation with Respect to Time}

Let us consider the discretization of Cauchy problem (6) with respect to a time variable. Let us assume that $\bar{\omega}_{\tau}=\left\{t_{n}=n \tau, n=0,1,2, \ldots\right\}$ is a net on interval $t \in[0, T]$ (a uniform net, for simplification). For any interval $\left(t_{n}, t_{n+1}\right) \in[0, T]$, the following identical equation from (6) is true:

$$
\int_{t_{n}}^{t_{n+1}}\left(-D \dot{u}_{h} \dot{\theta}+A u_{h} \theta\right) d t+\left.D \dot{u}_{h} \theta\right|_{t_{n}} ^{t_{n+1}}=\int_{t_{n}}^{t_{n+1}} f(t) \theta(t) d t, \quad \forall \theta(t) \in H_{h}
$$

The approximate solution of problem (6) is assumed to be expressed as a third degree Hermite spline, as in [6]:

$$
u_{h}(t) \approx y(t)=y^{n} \phi_{00}^{n}(t)+\dot{y}^{n} \phi_{10}^{n}(t)+y^{n+1} \phi_{01}^{n}(t)+\dot{y}^{n+1} \phi_{11}^{n}(t),
$$

where

$$
\begin{gathered}
y^{n}=y\left(t_{n}\right), \dot{y}^{n}=\frac{d y}{d t}\left(t_{n}\right), \phi_{00}^{n}(t)=2 \xi^{3}-3 \xi^{2}+1, \phi_{01}^{n}(t)=3 \xi^{2}+2 \xi^{3}, \\
\phi_{10}^{n}(t)=\tau\left(\xi^{3}-2 \xi^{2}+\xi\right), \phi_{11}^{n}(t)=\tau\left(\xi^{3}-\xi^{2}\right), \xi=\left(t-t_{n}\right) / \tau . \\
y^{n}=y\left(t_{n}\right), \dot{y}^{n}=\frac{d y}{d t}\left(t_{n}\right), \phi_{00}^{n}(t)=2 \xi^{3}-3 \xi^{2}+1, \phi_{01}^{n}(t)=3 \xi^{2}+2 \xi^{3}, \\
\phi_{10}^{n}(t)=\tau\left(\xi^{3}-2 \xi^{2}+\xi\right), \phi_{11}^{n}(t)=\tau\left(\xi^{3}-\xi^{2}\right), \xi=\left(t-t_{n}\right) / \tau .
\end{gathered}
$$

Selecting different weighting functions $\theta(t)$, specifically

$$
\theta_{1}(\xi)=0.5, \theta_{2}(\xi)=s_{1} \tau(\xi-0.5)+s_{2} \tau\left(\xi^{3}-1.5 \xi^{2}+0.5 \xi\right),
$$

where parameters are defined as $s_{1}=180 \beta-40 \alpha, s_{2}=1680 \beta-280 \alpha$, it is possible to obtain from (8) the following two-parameter vector difference scheme for unknown variables $\hat{y}, \hat{y}$ : 


$$
\begin{aligned}
& \left(D-\gamma \tau^{2} A\right) \frac{\hat{\dot{y}}-\dot{y}}{\tau}+A \frac{\hat{y}+y}{2}=\phi_{1}, \\
& \left(D-\alpha \tau^{2} A\right) \frac{\hat{y}-y}{\tau}-\left(D-\beta \tau^{2} A\right) \frac{\hat{\dot{y}}+\dot{y}}{2}=\phi_{2}, \\
& y^{0}=u_{0, h}, \dot{y}^{0}=u_{1, h} .
\end{aligned}
$$

where

$$
\hat{y}=y^{n+1}, \hat{\dot{y}}=\dot{y}^{n+1}, \gamma=1 / 12, \quad \phi_{k}=\int_{0}^{1} f\left(t_{n}+\tau \xi\right) \vartheta_{k}(\xi) d \xi, \quad k=1.2 .
$$

Parameters $\alpha, \beta$ are subject to the condition of the fourth-order approximation for scheme (9) [6]:

$$
\alpha-\beta=1 / 12
$$

Hereinafter, for definiteness, $\alpha=1 / 8, \beta=1 / 24$.

The methods for determining solutions of the Cauchy problem with respect to second-order equations, including solutions with improved accuracy, were also proposed in $[7,8$, and 10$]$.

\section{$4 \quad$ Stability and Convergence}

Theorem 1. [6, 10-14]. If $A^{*}=A \geq 0, D^{*}=D \geq 0$ and

$$
\alpha-\beta=1 / 12, D-\delta \tau^{2} A \geq \mu D, \forall \mu \in(0,1), \delta=\max \{\alpha, \beta, 1 / 12\}
$$

solution $y(t)$ for scheme (9) converges to the solution of problem (5), $u_{h}(t) \in C^{6}[0, T]$, and the following estimate is true:

$$
\left\|u_{h}(t)-y(t)\right\|_{A}+\left\|\dot{u}_{h}(t)-\dot{y}(t)\right\|_{D} \leq M \tau^{4} .
$$

The theorem proving is based on reducing two-layer vector scheme (9) to three-layer vector scheme separately for solution $y$ and its derivative $\dot{y}$. Constraint (11) for the values of parameters $\alpha=1 / 8, \beta=1 / 24$, which are in agreement with $\delta=1 / 8$, causes the following time constraint for a step:

$$
\tau \leq \frac{2 \sqrt{2}}{(1-\mu) \max \left(\omega_{0}, \alpha\right)}, \quad 0<\mu<1
$$

To estimate the accuracy of scheme (10), it is necessary to estimate error $z=u_{h}-u$. Using the method of such estimation according to the difference scheme theory [9], the final result shall be determined.

Theorem 2. If stability condition (12) for scheme (10) is met, solution $y(t)$ converges to a sufficiently smooth solution of problem (1-3), and the following estimate is true:

$$
\|u(t)-y(t)\|_{A}+\|\dot{u}(t)-\dot{y}(t)\|_{D} \leq M\left(h_{1}^{2}+h_{3}^{2}+\tau^{4}\right) .
$$

The fourth-order accuracy for a time step is possible due to the improved accuracy of a cubic spline. For the spatial step, a bilinear spline is selected, which ensures the accuracy for steps $h_{1}, h_{3}$ not higher than the second order.

\section{$5 \quad$ Algorithm for Scheme Implementation}

To implement scheme (9), it is necessary to solve the following system of two equations with respect to unknown variables $\hat{y}, \hat{\dot{y}}$ :

$$
m_{11} \hat{\dot{y}}+m_{12} \hat{y}=\Phi_{1}, \quad m_{21} \hat{\dot{y}}+m_{22} \hat{y}=\Phi_{2}
$$


where $m_{11}=D-\tau^{2} \gamma A, m_{12}=\frac{\tau}{2} A, m_{21}=-\frac{\tau}{2}\left(D-\beta \tau^{2} A\right), m_{22}=D-\alpha \tau^{2} A$,

$$
\Phi_{1}=\tau \phi_{1}-\frac{\tau}{2} A y+\left(D-\tau^{2} \gamma A\right) \dot{y}, \quad \Phi_{2}=\tau \phi_{2}+\left(D-\alpha \tau^{2} A\right) y+\frac{\tau}{2}\left(D-\beta \tau^{2} A\right) \dot{y}
$$

Excluding one of two unknown variables $\hat{\dot{y}}, \hat{y}$, two following equations can be obtained:

$$
C y^{n+1}=F_{1}, C \dot{y}^{n+1}=F_{2}
$$

where

$$
\begin{gathered}
C=m_{22} m_{11}-m_{12} m_{21}=D^{2}-\left(\alpha+\gamma-\frac{1}{4}\right) \tau^{2} A D+\left(\alpha \gamma-\frac{\beta}{4}\right) \tau^{4} A^{2}, \\
F_{1}=m_{22} \Phi_{1}-m_{12} \Phi_{2}, F_{2}=m_{11} \Phi_{2}-m_{21} \Phi_{1} .
\end{gathered}
$$

Matrix array $C$ is factorized as $C=C_{1} C_{2}=\left(D-\sigma_{1} A\right)\left(D-\sigma_{2} A\right)$, where $\sigma_{1}, \sigma_{2}$ are the roots of the following equation:

$$
\sigma^{2}+(\alpha+\gamma-1 / 4) \sigma+(\alpha \gamma-\beta / 4)=0 .
$$

For $\alpha=1 / 8, \beta=1 / 24$, for example, $\sigma_{1}=-1 / 24, \sigma_{2}=0$. In this case $y^{n+1}=C^{-1} F_{1}=C_{2}^{-1} C_{1}^{-1} F_{1}$, $\dot{y}^{n+1}=C_{2}^{-1} C_{1}^{-1} F_{2}$.

Due to the degeneration of operators $A, D$, there is a problem associated with the algorithm for scheme (9) implementation, as operators $C_{1}, C_{2}$ are also degenerate operators.

For analyzing this problem and determining a method for eliminating the problem, let us use the method of separation of variables with respect to the problem (1-3). The solution of the boundary value problem can be presented as a Fourier series:

$$
u(x, t)=u\left(x_{1}, x_{3}, t\right)=\sum_{n_{1}=0}^{\infty} \sum_{n_{3}=0}^{\infty} U\left(t ; n_{1}, n_{3}\right) X_{1}\left(x_{1} ; n_{1}\right) X_{3}\left(x_{3} ; n_{3}\right)
$$

where

$$
X_{k}^{\prime \prime}\left(x_{k}\right)=\lambda_{k}\left(n_{k}\right) X_{k}\left(x_{k}\right), \lambda_{k}\left(n_{k}\right)=\left(\frac{n_{k} \pi}{l_{k}}\right)^{2}, n_{k}=\overline{0, \infty}, X_{k}\left(x_{k} ; n_{k}\right)=\cos \frac{n_{k} \pi x_{k}}{l_{k}}, k=1.3 .
$$

The Fourier constants satisfy the following equation:

$$
\left(\lambda_{1}\left(n_{1}\right)+\lambda_{3}\left(n_{3}\right)\right) \frac{d^{2} U\left(t ; n_{1}, n_{3}\right)}{d t^{2}}+\left(\omega_{0}^{2} \lambda_{1}\left(n_{1}\right)+\alpha^{2} \lambda_{3}\left(n_{3}\right)\right) U\left(t ; n_{1}, n_{3}\right)=\Phi\left(t ; n_{1}, n_{3}\right)
$$

where

$$
\Phi\left(t ; n_{1}, n_{3}\right)=\int_{0}^{h_{1}} \int_{0}^{l_{3}} f\left(x_{1}, x_{3}, t\right) X_{1}\left(x_{1} ; n_{1}\right) X_{3}\left(x_{3} ; n_{3}\right) d x_{1} d x_{3} .
$$

As $\lambda_{1}(0)=0, \lambda_{3}(0)=0, X_{1}(0)=1, X_{3}(0)=0$, coefficient $U(t ; 0,0)$ can be determined from the following incompatible equation:

$$
0 \equiv \frac{d^{2} U(t ; 0,0)}{d t^{2}}(0)+\left(\omega_{0}^{2} \cdot 0+\alpha^{2} \cdot 0\right) U(t ; 0,0)=\Phi(t ; 0,0) \neq 0
$$

To eliminate the problem associated with degeneration of operators $A=A^{*}, D=D^{*}$, let us use the regularization principle, which, for self-conjugate operators, allows the operators to be displaced over spectrum [15] as $\tilde{A}=A+\varepsilon E, \tilde{D}=D+\varepsilon E$, where $\varepsilon>0$ is a small parameter which characterizes the value of the displacement of the operators over the spectrum.

As a result, scheme (9) can be replaced with the following regularized scheme: 


$$
\begin{gathered}
\left(\tilde{D}-\gamma \tau^{2} \tilde{A}\right) \frac{\hat{\tilde{y}}-\tilde{\dot{y}}}{\tau}+\tilde{A} \frac{\hat{\tilde{y}}+\tilde{y}}{2}=\phi_{1}, \\
\left(\tilde{D}-\alpha \tau^{2} \tilde{A}\right) \frac{\hat{\tilde{y}}-\tilde{y}}{\tau}-\left(\tilde{D}-\beta \tau^{2} \tilde{A}\right) \frac{\hat{\tilde{y}}+\tilde{\dot{y}}}{2}=\phi_{2}, \\
\tilde{y}^{0}=u_{0, h}, \tilde{\dot{y}}^{0}=u_{1, h} .
\end{gathered}
$$

Operators $A, D$ in the implementation algorithm are also replaced with operators $\tilde{A}, \tilde{D}$.

Instead of equation (16), the following equation for harmonic components can be obtained:

$$
\frac{d^{2} \tilde{U}\left(t ; n_{1}, n_{3}\right)}{d t^{2}}\left(\tilde{\lambda}_{1}\left(n_{1}\right)+\tilde{\lambda}_{3}\left(n_{3}\right)\right)+\left(\omega_{0}^{2} \tilde{\lambda}_{1}\left(n_{1}\right)+\alpha^{2} \tilde{\lambda}_{3}\left(n_{3}\right)\right) \tilde{U}\left(t ; n_{1}, n_{3}\right)=\Phi\left(t ; n_{1}, n_{3}\right) .
$$

For $n_{1}=0, n_{3}=0$ the following nondegenerate equation for harmonic component $\tilde{U}(t ; 0,0)$ can be obtained:

$$
\frac{d^{2} \tilde{U}(t ; 0,0)}{d t^{2}}+\tilde{U}(t ; 0,0)=\Phi(t ; 0,0) / \varepsilon
$$

It is evident that this harmonic component is factious (nonphysical), as value $\varepsilon$ is arbitrary. Taking into account that the solution of equation (1) appears as velocity potential $\vec{v}=\left(\frac{\partial u}{\partial x_{3}},-\frac{\partial u}{\partial x_{1}}\right)$, harmonic component $\tilde{U}(t ; 0,0)$ disappears in differentiation with respect to spatial variables. The error in restoring other harmonic components is insignificant if $\varepsilon<<\max \left(\omega_{0}, \alpha\right)$.

To inverse matrix array $\tilde{C}_{1}, \tilde{C}_{2}$, the direct square-root method was used once at the initial instant of time. The solution for the other layers was determined by multiplying matrix array $\tilde{C}^{-1}=\tilde{C}_{2}^{-1} \tilde{C}_{1}^{-1}$ by vector $F_{1}, F_{2}$.

Note. The proposed method for solving problem (1-3) can be used in the case of variable parameters $\omega_{0}\left(x_{3}\right), \alpha\left(x_{3}\right)$.

\section{Validation of the Algorithm for Solving the Second-Order Scalar Equation}

To check the accuracy of the in-time solution for finite element scheme (9), let us consider the model of Cauchy problem for the following scalar equation:

$$
\frac{d^{2} u}{d t^{2}}+v_{0}^{2} u=f(t), u(0)=u_{0}, \frac{d u}{d t}(0)=u_{1}
$$

In the case when $f(t)=A \sin \omega t$, where $\omega=v_{0}$ (resonance state), the solution of the equation is the following:

$$
u(t)=-0.5 t v_{0}^{-1} \cos \left(v_{0} t\right)
$$

The initial conditions corresponding to this solution are $u(0)=0, \dot{u}(0)=-0.5 v_{0}^{-1}$.

Let us consider problem (20) with a right-hand member:

$$
f(t)= \begin{cases}A \sin \omega t, & 0<t \leq t_{0}, \\ 0, & t>t_{0},\end{cases}
$$

that is, when an exciting force does not act for $t>t_{0}$, and, as a result, oscillations continue mechanically. 
For $0<t \leq t_{0}$ the solution is determined from mathematical expression (21). For $t>t_{0}$ the solution is the following:

$$
u(t)=\bar{u}_{0} \cos \left(v_{0}\left(t-t_{0}\right)\right)+\frac{\bar{u}_{1}}{v_{0}} \sin \left(v_{0}\left(t-t_{0}\right)\right)
$$

where $\bar{u}_{0}=-t_{0} \cos v_{0} t_{0} / 2 \omega_{0}, \bar{u}_{1}=-\left(\cos v_{0} t_{0}-t_{0} \sin v_{0} t_{0}\right) / 2 v_{0}$. The characteristic of the solution for $\omega=v_{0}=2, \quad t_{0}=3 \pi$ is shown in Figure 1.

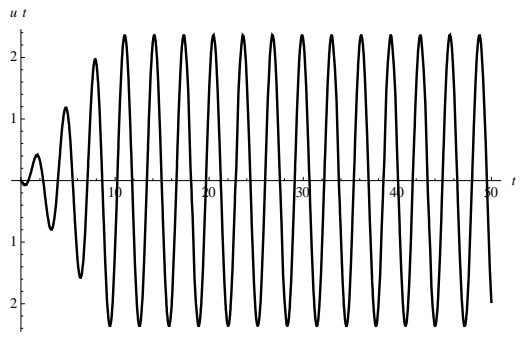

Figure 1. Characteristic of solution $u(t)$; mathematical expressions (2) and (3)

Let us compare errors of the solution of problem (20) for two numerical methods:

$>$ Errors for the method with second order accuracy:

$$
\begin{aligned}
& \frac{y_{n+1}-2 y_{n}+y_{n-1}}{\tau^{2}}+v_{0}^{2} y_{n}=f\left(t_{n}\right), n=1,2, \ldots \\
& y_{0}=u_{0}, y_{1}=u_{0}+\tau u_{1}+0.5 \tau^{2}\left(f\left(t_{0}\right)-v_{0}^{2} y_{0}\right) .
\end{aligned}
$$

Errors for the scalar analogue of method (9) with fourth order accuracy

$$
\left\{\begin{array}{l}
\left(1-\gamma \tau^{2} v_{0}^{2}\right) \frac{\dot{y}_{n+1}-\dot{y}_{n}}{\tau}+v_{0}^{2} \frac{\dot{y}_{n+1}+\dot{y}_{n}}{2}=\phi_{1}, \quad n=0,1,2, \ldots \\
\left(1-\alpha \tau^{2} v_{0}^{2}\right) \frac{y_{n+1}-y_{n}}{\tau}-\left(1-\beta \tau^{2} v_{0}^{2}\right) \frac{\dot{y}_{n+1}+\dot{y}_{n+1}}{2}=\phi_{2}, y_{0}=u_{0}, \dot{y}_{0}=u_{1} .
\end{array}\right.
$$

The characteristics of errors of the methods are shown in Figure 2 (method 23) and Figure 3 (method 24).

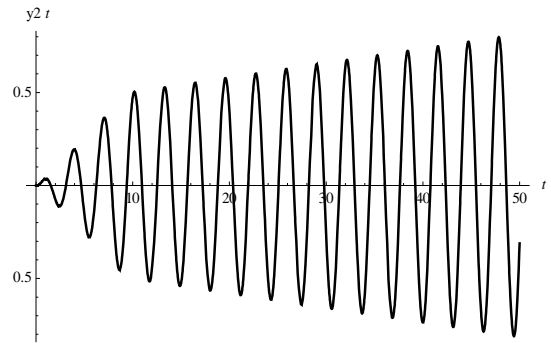

Figure 2. Error of method (23):

$$
\Delta y 2(t)=y 2(t)-u(t)
$$

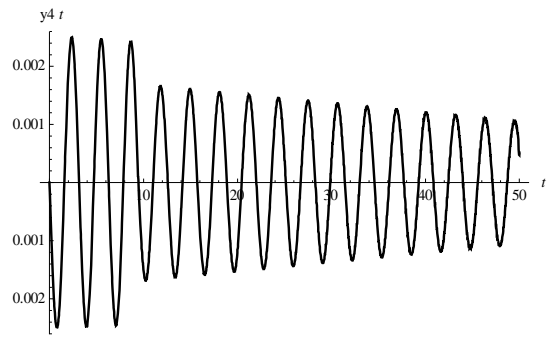

Figure 3. Error of method (24):

$$
\Delta y 4(t)=y 4(t)-u(t)
$$

The results of the analysis of the error characteristics demonstrate that the error of method (24) is lower by about three orders than the error of method (23).

The characteristics of solution $u(t)$ of the differential problem (blue color) and approximate solution $y 2(t)$ (black color) obtained by using the method with second order accuracy are shown in Figure 4. The characteristic of solution $y 4(t)$ obtained by using method (19) with fourth order accuracy virtually coincides with the characteristic of exact solution $u(t)$ and for this reason is not presented. 


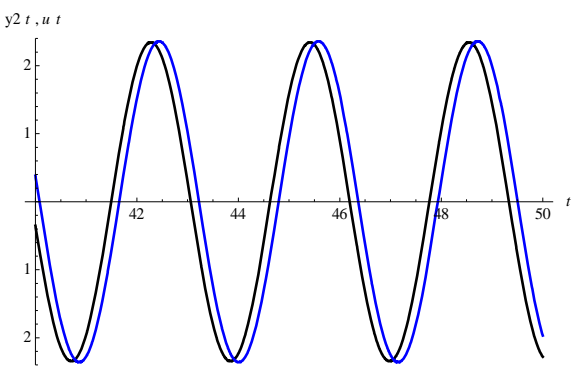

Figure 4. Characteristics of solution $u(t)$ (blue color) and solution $y 2(t)$ (black color)

\section{Computational Experiments}

The computational experiments with respect to the solution of problem (1-3) are described below. The factor which excites motions of liquid is represented by the right-hand member of equation (1) which is effective within section $2 \Delta$ in length along axis $x_{3} \in\left[0, l_{3}\right],\left(\Delta<<l_{1}\right)[6]$ :

$$
\begin{gathered}
f\left(x_{1}, x_{3}, t\right)=\varphi\left(x_{1}, x_{3}\right) \phi(t), \\
\varphi\left(x_{1}, x_{3}\right)= \begin{cases}1, & \left|x_{1}-l_{1} / 2\right| \leq \Delta, x_{3}=l_{3} / 2, \\
0, & \text { otherwise }\end{cases}
\end{gathered}
$$

and within finite interval of time:

$$
\phi(t)= \begin{cases}\sin (\omega t), & 0 \leq t \leq t_{0}, \\ 0, & t>t_{0}\end{cases}
$$

The initial conditions are assumed to be uniform:

$$
u_{0}(x)=u_{1}(x)=0 .
$$

The dimensions of area $\left(x_{1}, x_{3}\right) \in \Omega$ are $l_{1}=l_{2}=10, \Delta=0.1$ and $0 \leq t \leq 40$. The net parameters are the following:

$$
h_{1}=h_{3}=0.3125, \quad N_{1}=N_{3}=32, \quad \tau=.1
$$

The characteristic of function $\varphi\left(x_{1}, x_{3}\right)$ is shown in Figure 5. The scale of the characteristic corresponds to partitioning $N_{1}=N_{3}=32$.

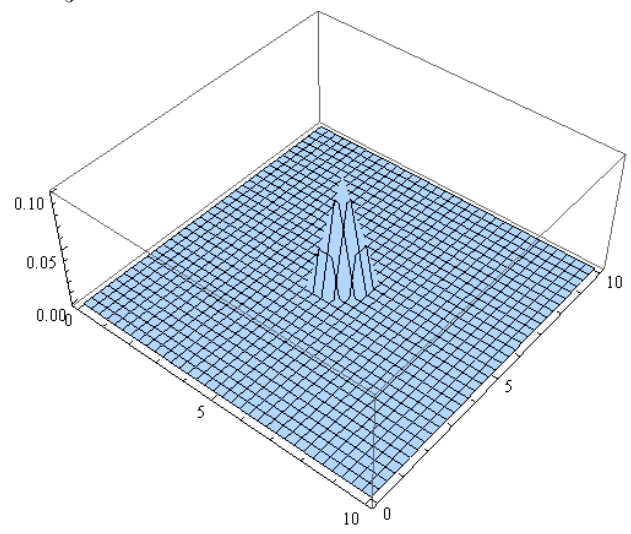

Figure 5. Characteristic $\varphi\left(x_{1}, x_{3}\right), \Delta=0.1$

The calculation results are presented in Figures 7-20. For all the variants, the regularization parameter is $\varepsilon=0.001$. 
Figure 6 shows characteristic $\phi(t)$ of the variation of amplitude $f\left(x_{1}, x_{1}, t\right)$ as a function of time at point $x_{1}=l_{1} / 2, x_{2}=l_{2} / 2$ for $\omega=1, t_{0}=6 \pi \approx 18.8496$.

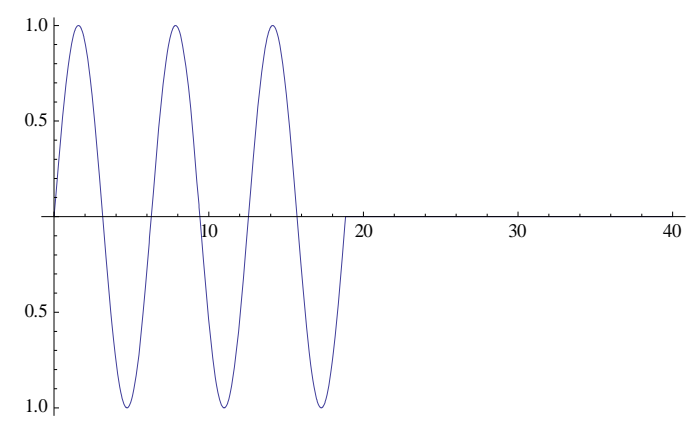

Figure 6. Characteristic $\phi(t)$

Figure 7 shows characteristic $V 0(t)=y\left(l_{1} / 2, l_{3} / 2, t\right)$ illustrating the change of solution $y\left(x_{1}, x_{3}, t\right)$ as a function of time at the midpoint of area $\Omega$ for $\omega_{0}=\pi, \omega=\alpha=1, t_{0}=6 \pi=18.8496$. The results of the computational experiments with different values of parameter $\varepsilon$ demonstrate the dependence of oscillation amplitude $V 0(t)$ on the value of this parameter. In such a case, the oscillation mode is not changed.

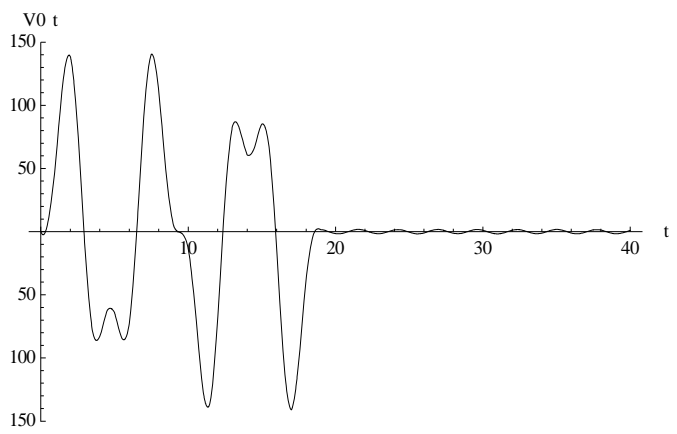

Figure 7. Characteristic $V 0(t)$ at $\omega=\alpha=1, \omega_{0}=\pi$.

Figure 8 shows the 3D characteristic of solution $Y\left(x_{1}, x_{3}, t\right)=y\left(x_{1}, x_{3}, t\right)-V 0(t)$. Figure 9 shows the characteristic of velocity $\vec{v}\left(x_{1}, x_{3}, t\right)=\left(\frac{\partial y}{\partial x_{3}},-\frac{\partial y}{\partial x_{1}}\right)$ at instant of time $t=36$.

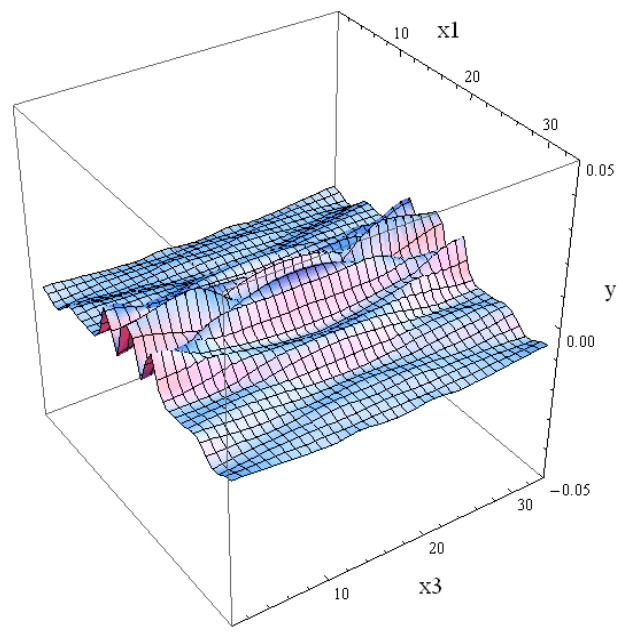

Figure 8. Characteristic $Y\left(x_{1}, x_{3}, t=36\right)$

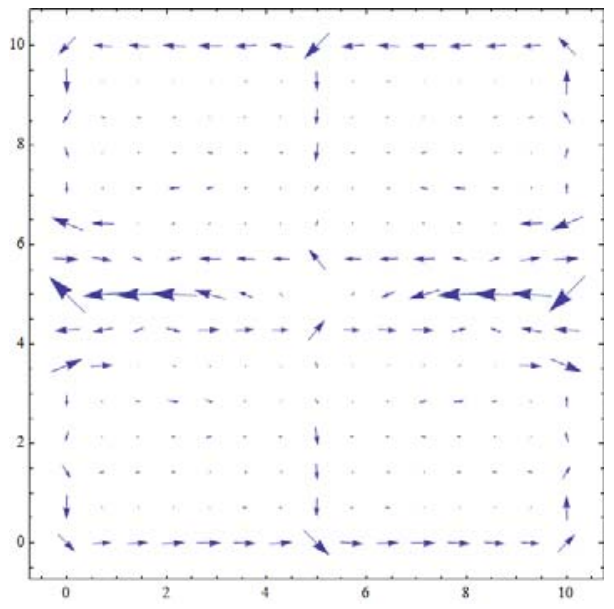

Figure 9. Characteristic of the field of velocities

$$
\vec{v}\left(x_{1}, x_{3}, t=36\right)
$$


To compare the solution of the Neumann problem with the solution of the Dirichlet problem, where $u=0, x \in \Gamma$ instead of (2), let us show the characteristics of the solution of the Dirichlet problem, with the same values of parameters as those for the Neumann problem, whose characteristics are shown in Figures 7, 8, and 9. See Figure $10-12$ for results. It should be noted that in the case of the Dirichlet problem, oscillations $V 0(t)$ at the midpoint of area $\Omega$ do not depend on the fictitious oscillations of the zero harmonic component of the solution, as in the case of the Neumann problem.

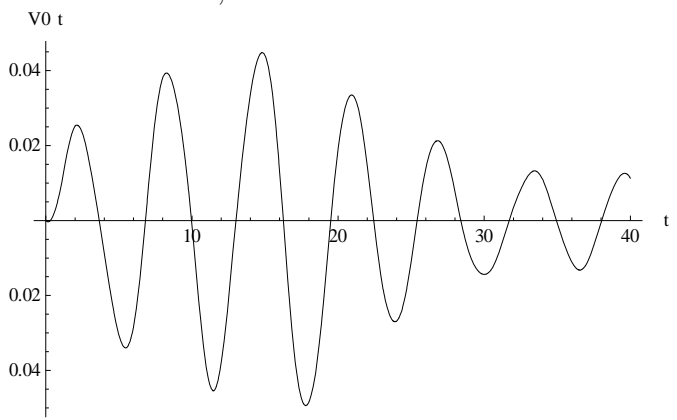

Figure 10. Characteristic $V 0(t)$ at $\omega=\alpha=1, \omega_{0}=\pi$.

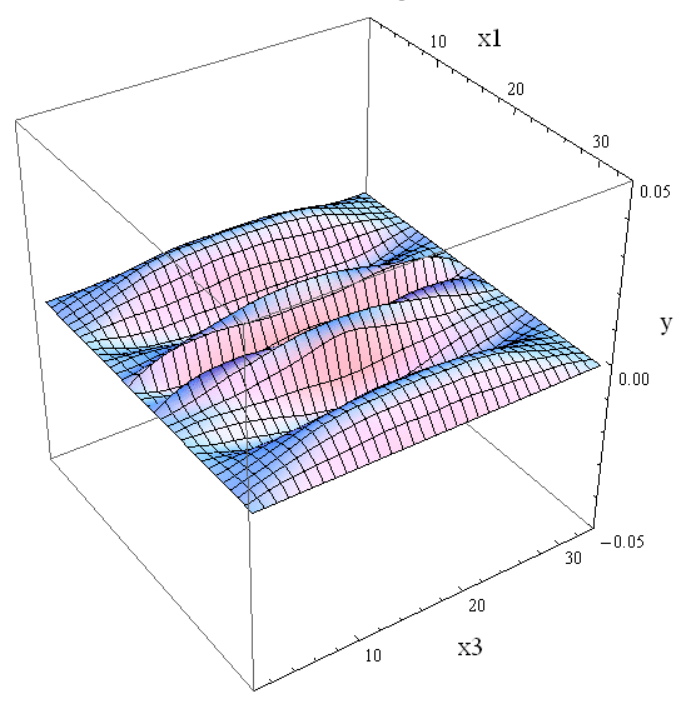

Figure 11. Characteristic $Y\left(x_{1}, x_{3}, t=36\right)$

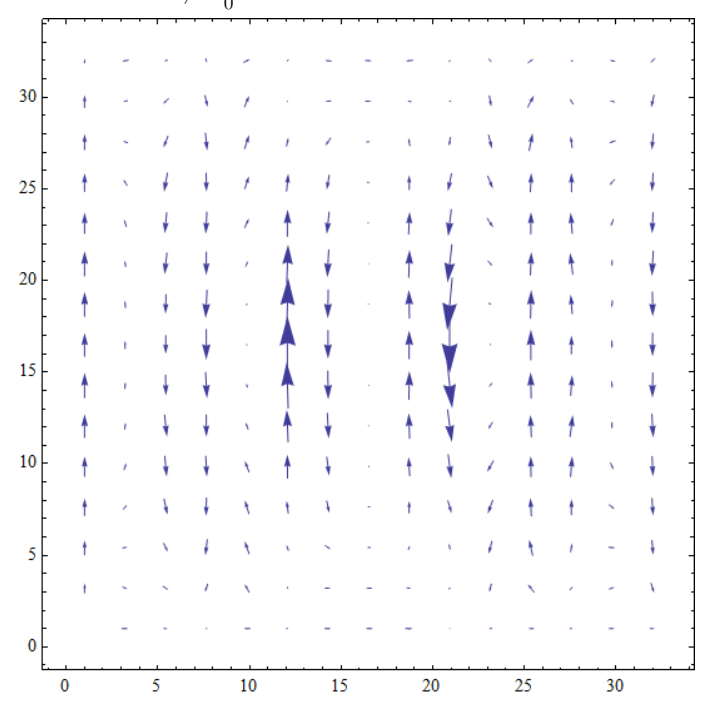

Figure 12. Characteristic of the field of velocities

$$
\vec{v}\left(x_{1}, x_{3}, t=36\right)
$$

The comparison of the results demonstrates the essential distinction between the solutions of Neumann problem and that of the Dirichlet problem.

Let us consider the Neumann problem at parameter values $\omega=\omega_{0}=\pi, \alpha=1, t_{0}=6$. See Figure $13-$ 16 for results.

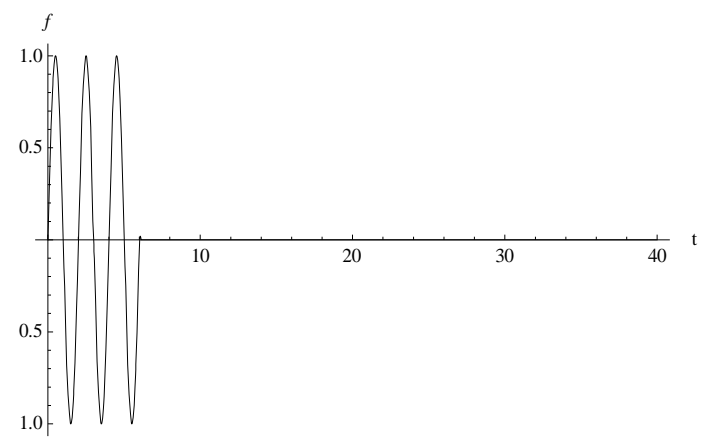

Figure 13. Characteristic $\phi(t)$ 


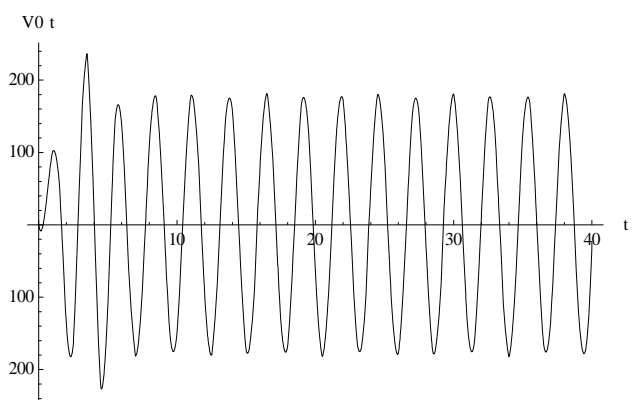

Figure 14. Characteristic $V 0(t)$ at $\omega=\omega_{0}=\pi, \alpha=1$.

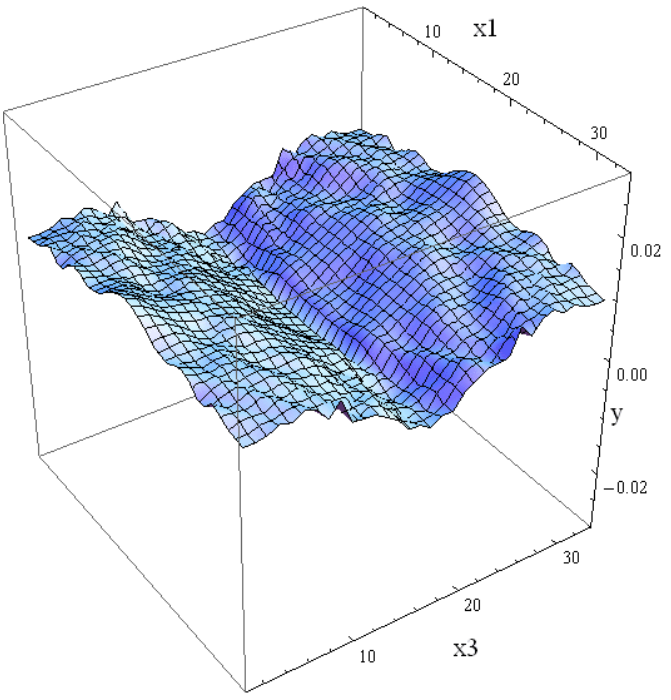

Figure 15. Characteristic $Y\left(x_{1}, x_{3}, t=40\right)$

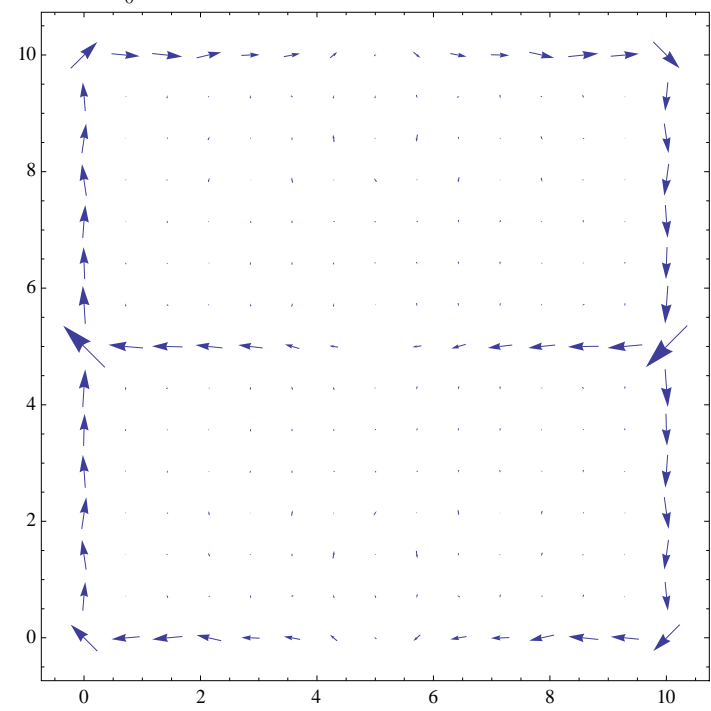

Figure 16. Characteristic $\vec{v}\left(x_{1}, x_{3}, t=40\right)$

Finally, let us consider the Neumann problem at parameter values $\omega=(1+\pi) / 2=2.07080$, $t_{0}=6 \pi / \omega=9.10256, \omega_{0}=\pi, \alpha=1$. See Figure $17-20$ for results.

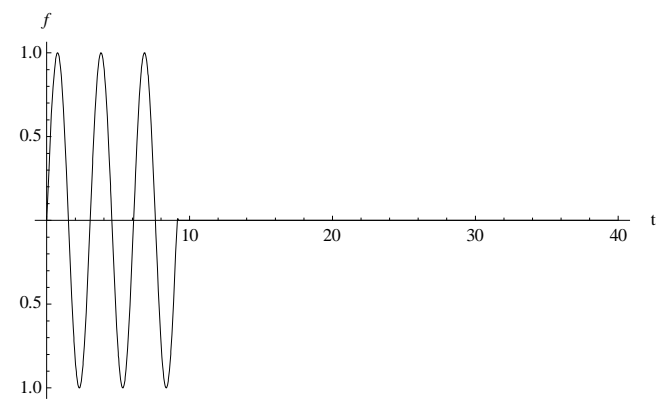

Figure 17. Characteristic $\phi(t), \omega=2.07080, t_{0}=6 \pi / \omega \approx 9.10256$

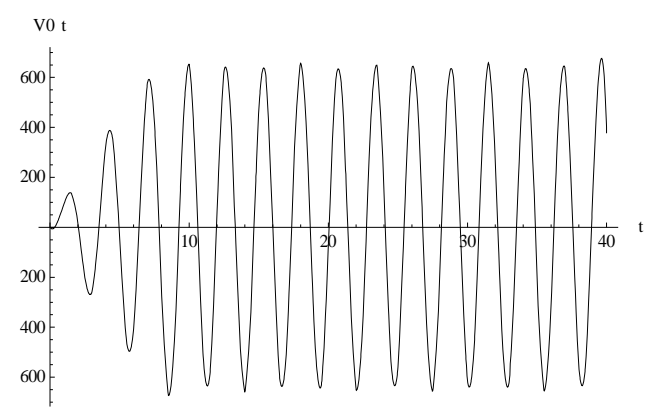

Figure 18. Characteristic $V 0(t) \quad \omega=2.07080, \omega_{0}=\pi, \alpha=1$ 


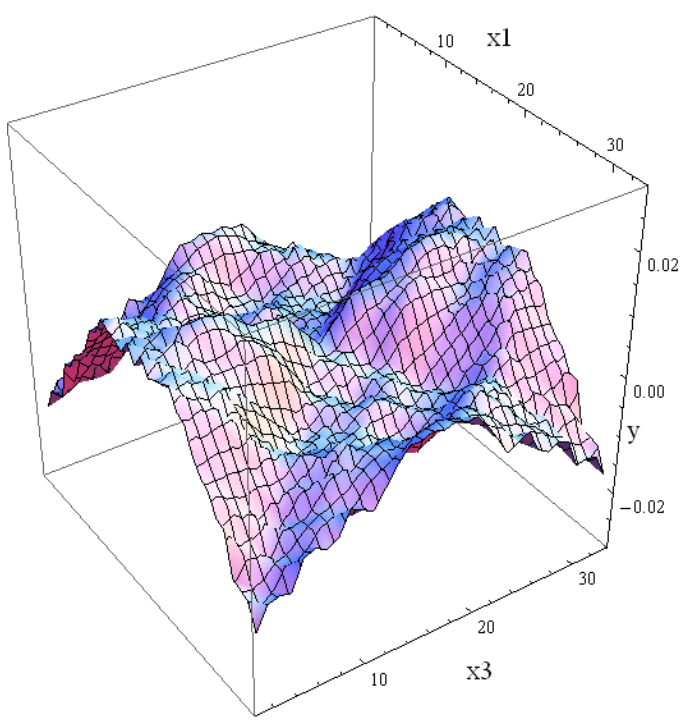

Figure 19. Characteristic $Y\left(x_{1}, x_{3}, t=38.5\right)$

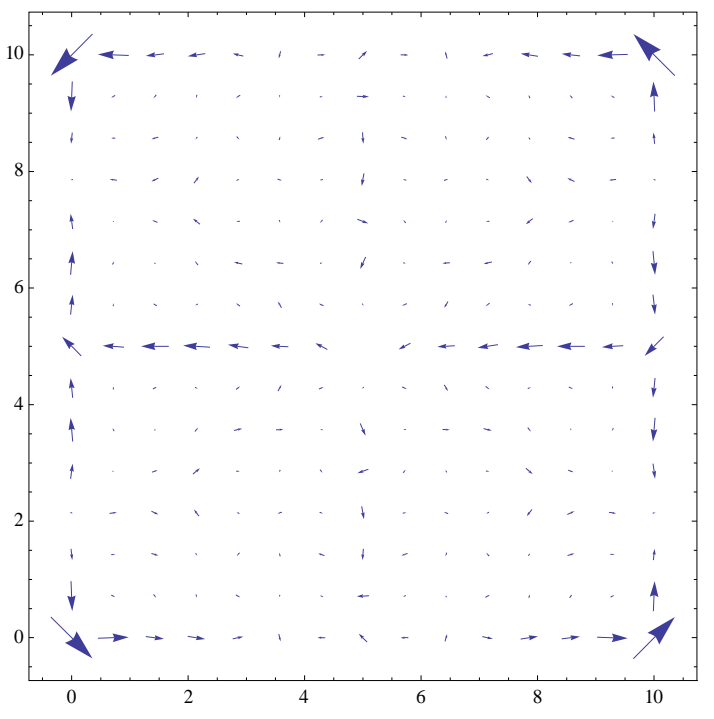

Figure 20. Characteristic $\vec{v}\left(x_{1}, x_{3}, t=38.5\right)$

\section{Conclusions}

The first conclusion coincides with the conclusion in our paper [6], that is, there is no decay of oscillations according to solution $Y\left(x_{1}, x_{3}, t\right)=y\left(x_{1}, x_{3}, t\right)-V 0(t)$ at $t \rightarrow \infty$.

As in [6], degeneration cases at $\omega=\omega_{0}$ and $\omega=\alpha$ cause solutions oriented in the direction of the corresponding coordinate (see Figures 8 and 15). At $\alpha<\omega<\omega_{0}$ the solution is changed both in direction $o x_{1}$ and in direction $o x_{3}$ (see Figure 19).

Accordingly, the developed procedure for solving the equation for gravity-gyroscopic waves provides the possibility to efficiently solve various initial boundary value problems, describing motions of liquid, according to the Bussinesk approximation.

\section{References}

1. S.A. Gabov and A.G. Sveshnikov, Linear Problems in the Theory of Non-Steady-State Internal Waves, Nauka, Moscow, 1990.

2. S.A. Gabov and Y.D. Pletner, "On an Initial Boundary Value Problem with Respect to the Equation for Gravity-Gyroscopic Waves," Journal of Computational Mathematics and Mathematical Physics, vol. 25, no.11, pp. 1689-1696, 1985.

3. S.A. Gabov and Y.D. Pletner, "Equation for Gravity-Gyroscopic Waves: Annular Potential and its Application," Journal of Computational Mathematics and Mathematical Physics, vol. 27, no. 1, pp. 102-113, 1997.

4. G.B. Whitham, Linear and Nonlinear Waves. Wiley, Hoboken. 1974.

5. Y.Z. Miropolskiy, Dynamics of Internal Waves in the Ocean, Gidrometeoizdat, Moscow, 1981.

6. M.N. Moskalkov and D. Utebaev, "Finite Element Solution of a Problem for Gravity Gyroscopic Equation in the Time Domain," Applied Mathematics, vol. 5, no. 8, pp. 1120-1132, 2014. http://dx.doi.org/10.4236/am.2014.58105

7. O.C. Zienkiewicz and K. Morgan, Finite Elements and Approximation, Wiley, Hoboken, 1983.

8. O.C. Zienkiewicz and R.L. Taylor, The Finite Element Method, Butterworth-Heinemann, 2000.

9. A.A. Samarskii, Theory of Difference Schemes, Nauka, Moskow, 1977.

10.M.N. Moskalkov, "Scheme of the High-Accuracy Finite Element Method for Solving Non-Steady-State SecondOrder Equations," Differential Equations, vol. 16, no. 7, pp. 1283-1292, 1980. 
11.M.N. Moskalkov and D. Utebaev, "Investigation of difference schemes of finite element method for second-order unsteady-state equations," Journal Computation Applied Mathematics, Kiev, no. 92, pp. 70 - 76. 2005.

12.M.N. Moskalkov and D. Utebaev, "Comparison of Some Methods for Solving the Internal Wave Propagation Problem in a Weakly Stratified Fluid," Mathematical Models and Computer Simulations. vol. 3, no. 2, pp. 264271, 2012. http://dx.doi.org/10.1134/S2070048211020086

13.M.N. Moskalkov and D. Utebaev, "Finite Element Method for the Gravity-Gyroscopic Wave Equation," Journal Computation and Applied Mathematics, no. 2(101), pp. 97 - 104, 2010.

14.M.N. Moskalkov and D. Utebaev, "Convergence of the Finite Element Scheme for the Equation of Internal Waves," Cybernetics and Systems Analysis, vol. 47, no. 3, pp. 459-465. http://dx.doi.org/10.1007/s10559-0119327-1

15.A.N. Tikhonov and V.Y. Arsenin, Methods for Solving Incorrect Problems, Nauka, Moscow, 1986. 